Algebraic $8 \mathcal{G}$ Geometric $\mathcal{T}$ opology

Volume 5 (2005) 107-118

Published: 7 February 2005

ATG

\title{
The Kauffman bracket skein module of a twist knot exterior
}

\author{
Doug Bullock \\ WALTER LO FARO
}

\begin{abstract}
We compute the Kauffman bracket skein module of the complement of a twist knot, finding that it is free and infinite dimensional. The basis consists of cables of a two-component link, one component of which is a meridian of the knot. The cabling of the meridian can be arbitrarily large while the cabling of the other component is limited to the number of twists.
\end{abstract}

AMS Classification 57M27; 57M99

Keywords Knot, link, skein module, Kauffman bracket

\section{Introduction}

At first glance, and in original intent [13], the Kauffman bracket skein module is a formal extension of the Kauffman bracket polynomial to an arbitrary 3manifold. As Kauffman's polynomial (for framed links in $S^{3}$ ) is equivalent to the Jones polynomial (for oriented links in $S^{3}$ ), one may think of the skein module as a generalization of the Jones polynomial. More recently the module has taken on a different significance: it is now seen as a deformation of the $S L_{2}(\mathbb{C})$-characters of the fundamental group [4, 5, 14. Using this interpretation of the skein module of a knot exterior, Frohman, Gelca and the second author here constructed a quantum version the $A$-polynomial [6]. This is related back to the Jones polynomial [15, 16] (not simply by generality) and has implications for the hyperbolic volume conjecture [9, 12. Despite all this, there have as yet been no computations of Kauffman bracket skein modules for hyperbolic manifolds.

Early computations [13] depended on an $I$-bundle structure for the manifold, since projection along the $I$ factor gave a natural mechanism for controlling complexity. The only other successful method [1, 7, 8, has been to consider the 
effect of adding a single 2-handle to a handlebody. This creates a presentation with fairly simple generators (any basis for the module of the handlebody), but having an unwieldy set of relations. Eliminating redundant relations is the most difficult part of the task. What is needed is an effective method of creating relations among relations, or syzygies, and then keeping track of which relations can be removed.

This has been managed for all genus one manifolds [7, 8], and for $(2, q)$-torus knot exteriors [1]. In principle, the combinatorics ought to be accessible for genus two manifolds with toral boundary (one added handle), but the computations are quite daunting in practice. Even for $(2, q)$-torus knots, the trick was managed only with help from a particularly nice basis.

The innovation in this paper is a simpler method of keeping track of the relations. We use the established handle addition technique, but we twist the handlebody instead of the handle, which simplifies many bracket computations. Our viewpoint also leads to a comfortable and practical method for producing syzygies that reduce the initial presentation to a simple basis.

\section{The theorem}

Let $M$ be an orientable 3-manifold. A framed link in $M$ is an embedding of a disjoint collection of annuli into $M$. Framed links are depicted by link diagrams showing the cores of an annuli lying flat in the projection plane (i.e. with blackboard framing).

Two framed links in $M$ are equivalent if there is an isotopy of $M$ taking one to the other. Let $\mathcal{L}_{M}$ denote the set of equivalence classes of framed links in $M$, including the empty link. With $R=\mathbb{Z}\left[t^{ \pm 1}\right]$, form a free module $R \mathcal{L}_{M}$ with basis $\mathcal{L}_{M}$. Define $S(M)$ to be the smallest submodule of $R \mathcal{L}_{M}$ containing

all expressions of the form $\left.\backslash-t \smile-t^{-1}\right)\left(\right.$ and $\bigcirc+t^{2}+t^{-2}$, where the framed links in each expression are identical outside balls pictured in the diagrams. The Kauffman bracket skein module $K(M)$ is the quotient

$$
R \mathcal{L}_{M} / S(M)
$$

A $q$-twist knot (right-handed, if not amphichiral) is the alternating knot formed by inserting a left-to-right string of $q$ half-twists into the coupon in Figure 1. The 2-twist knot in Figure 2 2 for example, is the familiar figure-8 knot. Let $M_{q}$ be the twist knot exterior, and denote by $x y$ the 0 -framed, two component link also pictured in Figure 11. The meridian is $x$ and the other component is 


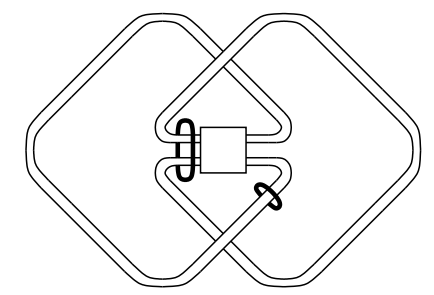

Figure 1: Exterior of a $q$-twist knot and the link $x y$

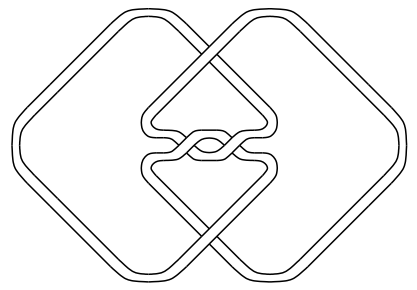

Figure 2: Figure-8 knot as a 2-twist knot

$y$. In general, $x^{l} y^{m}$ denotes the cable of $x y$ consisting of $l$ parallel copies of $x$ and $m$ parallel copies of $y$. The exponents run over non-negative integers and 1 denotes the empty link.

Theorem 2.1 $K\left(M_{q}\right)$ is free with basis $\left\{x^{l} y^{m} \mid m \leq q\right\}$

\section{Initial presentation}

The knot exterior $M_{q}$ decomposes into a pair of genus two handlebodies glued along the 4-punctured sphere $S$ shown in Figure 3 Let $H$ be the closure of the component of $M_{q}-S$ containing the coupon. Figure 4 (a) depicts $H$, slightly deformed so that the upper left and lower right punctures are in the foreground.

The portions of the knot outside $H$ are parallel to a pair of $\operatorname{arcs}$ in $S$ that cut it into an annulus. Therefore, $M_{q}$ is homeomorphic to $H$ with a 2-handle attached along this annulus. Its core is shown in Figure 廿(b).

There is a standard argument [1, 2, 17, 8, 10, that says $K\left(M_{q}\right)$ is $K(H)$ modulo skeins differing by slides across the 2-handle. We find this language to be a little imprecise, so we will rephrase it in terms of relative skeins. Suppose that the core of the attaching annulus is given the blackboard framing in $S$. We cut out a very small bit of this curve, as indicated in Figure $4(\mathrm{c})$, leaving a framed 


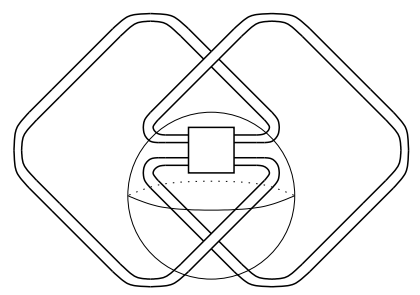

Figure 3: Decomposing sphere $S$ in $M_{q}$

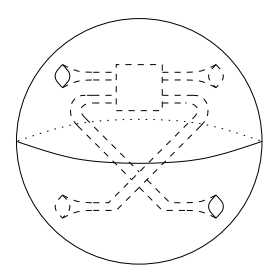

(a)

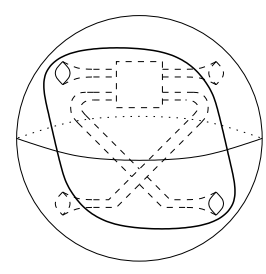

(b)

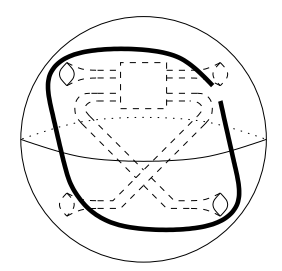

(c)

Figure 4: (a) $H$ (b) Core of the attaching annulus (c) The core as a relative link

arc whose ends are a pair of framed points in $\partial H$. Following [11, 13, let $K_{1}(H)$ be the skein module of $H$ relative to those two framed points.

Let $L$ be a relative link in $H$. Since the ends of $L$ are very close together we can unambiguously define the completion of $L$ to be the result of gluing its ends together. The slide of $L$ is formed by gluing its ends to the cut open core of the attaching annulus. Completion and slide are denoted by $c(L)$ and $s(L)$. Let $r(L)=c(L)-s(L)$ and extend linearly to $r: K_{1}(H) \rightarrow K(H)$. The image of $r$ is the set of all possible relations in $K(H)$ induced by handle slides. Therefore,

$$
K\left(M_{q}\right)=K(H) / r\left(K_{1}(H)\right) .
$$

Since $K(H)$ is free, the quotient provides a presentation of $K\left(M_{q}\right)$. Any basis for $K(H)$ serves as a generating set. For relations, choose generators for $K_{1}(H)$, apply $r$, and express everything in terms of the basis of $K(H)$. The more efficient your generating set for $K_{1}(H)$, the more efficient your presentation. However, even a basis for $K_{1}(H)$ yields unnecessary relations. Computing $K\left(M_{q}\right)$ thus becomes a search for all relations among the relations in this presentation.

We need to fix a basis for $K(H)$. Let $x$ and $y$ be the knots in Figure 1, but only up to isotopy in $H$. Let $z$ be a meridian that is not isotopic to $x$ in $H$. As usual, $x, y$ and $z$ are 0 -framed. The set of cables, $\mathcal{B}=\left\{x^{l} y^{m} z^{n}\right\}$, is a basis for $K(H)$ [13. 
We also need to fix generators of $K_{1}(H)$, but first some notes on multiplicative notation for (possibly relative) links in $H$.

- The notation is commutative and associative.

- $x^{l}, y^{m}$ and $z^{n}$ denote cables.

- If $L$ is a (possibly relative) link then $x^{l} L$ means the union of $L$, pushed away from the knot boundary, with a cable of $x$ very near the knot boundary.

- Similarly for $z^{n} L$.

- If $\sigma$ is a (possibly relative) skein then $x^{l} z^{n} \sigma$ is defined by distributing $x^{l} z^{n}$ across any linear combination of links representing $\sigma$. This is well defined because representatives of $\sigma$ differ by skein relations that take place away from the knot boundary.

- If $\sigma$ is written in terms of the basis $\left\{x^{i} y^{j} z^{k}\right\}$ then $x^{l} z^{n} \sigma$ is just polynomial multiplication.

- In general, $y^{m} L$ is not well defined, but there are some specific embeddings of $L$ for which we want $y^{m} L$ to make sense. These are explained below.

If $L$ is one of the relative knots

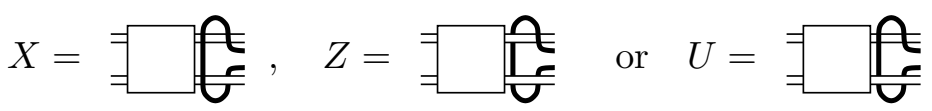

then $y^{m} L$ denotes a copy of $y^{m}$ inserted into the twist coupon. If $0 \leq k \leq q$, let

$$
Y_{k}=={ }^{a-k}{ }^{2}
$$

where the coupons contain $q-k$ and $k$ twists. By $y^{m} Y_{k}$ we mean a cable of $y$ inserted into the coupon containing $k$ twists, even if $k=0$. Lastly,

$$
y^{m} Y_{-1}=={ }_{q}
$$

with $y^{m}$ inserted in the coupon that contains no twists.

Lemma 3.1 $K_{1}(H)$ is generated by $\left\{x^{l} y^{m} z^{n} L \mid L=X, Y_{0}, Z\right.$, or $\left.U\right\} 1$

\footnotetext{
${ }^{1}$ It's actually a basis, but the proof is annoying and the result is unnecessary.
} 


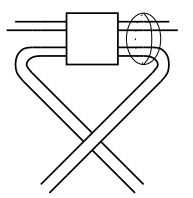

Figure 5

Proof Given any relative link in $H$, it can be isotoped into the oval neighborhood shown in Figure 5. Once there, grab the top and bottom of the oval and twist in opposite directions a quarter turn each. This should make the tubes perpendicular to the page so that

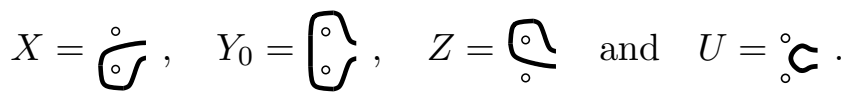

If not, twist the opposite way and it will.

Now resolve according to a relative version of the argument in [3, Lemmas 1-3]. Each term of the resolution will be a cable of

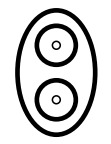

together with one of $X, Y_{0}, Z$ or $U$. The modification introduced in 14, Theorem 6.2] lets us force an $X$ to end up above the cabled link and a $Z$ to end up below it. Neither $Y_{0}$ nor $U$ can become entangled. Now we untwist the oval neighborhood, returning $X, Y_{0}, Z$ and $U$ to their initial embeddings. This will twist the cabled link, but it can be further resolved into a polynomial in $x, y$ and $z$.

\section{Sufficient relations}

In this section we locate in $r\left(K_{1}(H)\right)$ sufficient relations to eliminate all but $\left\{x^{l} y^{m} \mid m \leq q\right\}$ from $\mathcal{B}$. It turns out that powers of $z$ are easy to eliminate and that powers of $y$ index the complexity of other computations. For this reason, we introduce the notation $\sigma \sim y^{m}$, meaning $\sigma=t^{ \pm p} y^{m}$ modulo the span of $\left\{x^{i} y^{j} z^{k} \mid j<m\right\}$.

Where $x$ and $z$ are concerned, the relation submodule behaves like an ideal.

Lemma 4.1 If $\sigma \in r\left(K_{1}(H)\right)$, then $x^{i} z^{k} \sigma \in r\left(K_{1}(H)\right)$. 
Proof Suppose $L$ is a relative link in $H$. Since $x$ and $z$ are nowhere near the attaching annulus on $\partial H$, it's easy to see that $x^{i} z^{k} r(L)=r\left(x^{i} z^{k} L\right)$. This extends to all of $K_{1}(H)$.

In practice, you compute a relation by grinding some $r(\sigma)$ down to a polynomial in $x, y$ and $z$. Lemma 4.1 then says that any formal multiple of that relation by $x^{i} z^{k}$ is another relation. For example, from the relation $r\left(y^{m} Z\right)$ we obtain a class of relations:

$$
\begin{aligned}
x^{l-1} z^{n} r\left(y^{m} Z\right) & =x^{l-1} z^{n}\left(y^{m} z-y^{m} x\right) \\
& =x^{l-1} y^{m} z^{n+1}-x^{l} y^{m} z^{n}
\end{aligned}
$$

Relations (11) can be used to eliminate $\left\{x^{l} y^{m} z^{n} \mid n>0\right\}$ from the presentation of $K\left(M_{q}\right)$. Powers of $y$ are more troublesome. To eliminate $\left\{x^{l} y^{m} \mid y>q\right\}$, we need some new relations.

Lemma $4.2 c\left(y^{m} Y_{k}\right) \sim y^{m+k+1}$

Proof Induct on $k$. If $k=0$, we have

$$
c\left(y^{m} Y_{0}\right)=-t^{-3}
$$

By counting wrapping numbers in each term of the resolution one can see that $c\left(y^{m} Y_{0}\right) \sim y^{m+1}$.

Another wrapping number argument shows that no term of $c\left(y^{m} Y_{-1}\right)$ has a power of $y$ larger than $m$.

For $k \geq 1$ consider the relation

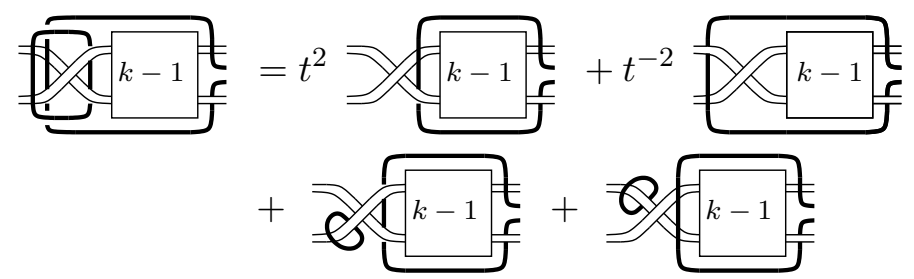

Insert $y^{m}$ into the coupon and take the closure of every term to get

$$
c\left(y^{m+1} Y_{k-1}\right)=t^{2} c\left(y^{m} Y_{k-2}\right)+t^{-2} c\left(y^{m} Y_{k}\right)+y^{m} \text { (meridians), }
$$

which can be solved for $y^{m} Y_{k}$. 
Lemma $4.3 s\left(y^{m} Y_{q}\right) \sim y^{q+m}$.

Proof Note first that

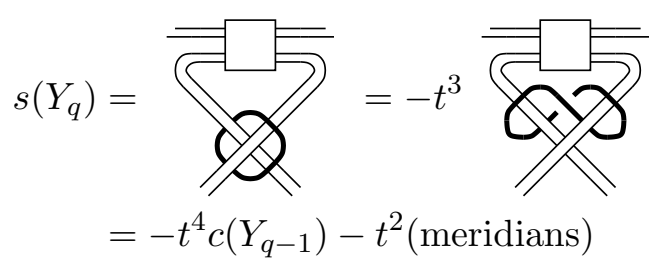

Then insert $y^{m}$ into the coupon and apply Lemma 4.2.

Lemmas 4.2 and 4.3 imply $r\left(y^{m} Y_{q}\right) \sim y^{q+m+1}$. Extended to include powers of $x$, there relations serve to eliminate any remaining terms of $\mathcal{B}$ with $y$-degree greater than $q$. Hence, with

$$
\mathcal{R}=\left\{x^{i} z^{k} r\left(y^{m} Z\right)\right\} \cup\left\{x^{i} r\left(y^{m} Y_{q}\right)\right\}
$$

Proposition 4.4 The presentation $\mathcal{B}$ modulo $\mathcal{R}$ reduces to the free presentation of Theorem 1.

To finish the proof of Theorem 1 we must find relations among the relations $r\left(K_{1}(H)\right)$ sufficient to write them all in terms of $\mathcal{R}$. Such a relation among relations is called a syzygy.

\section{$5 \quad$ Syzygies}

Here we show that $\mathcal{R}$ generates $r\left(K_{1}(H)\right)$. Recall that $\mathcal{R}$ contains relations of the form

$$
\begin{aligned}
& r\left(x^{l} y^{m} z^{n} Z\right), \text { and } \\
& r\left(x^{l} y^{m} Y_{q}\right)
\end{aligned}
$$

We need to show that the span of $\mathcal{R}$, denoted $\langle\mathcal{R}\rangle$, contains all relations of the form

$$
\begin{aligned}
& r\left(x^{l} y^{m} z^{n} X\right), \\
& r\left(x^{l} y^{m} z^{n} Y_{0}\right), \text { and } \\
& r\left(x^{l} y^{m} z^{n} U\right)
\end{aligned}
$$

Lemma 5.1 If $L$ is any link in $H$ (or any skein in $K(H)$ ), then $x L-z L \in\langle\mathcal{R}\rangle$. 
Proof Express $L$ in terms of the basis $\mathcal{B}$ and then apply (1).

Lemma $5.2 r\left(x^{l} y^{m} z^{n} X\right) \in\langle\mathcal{R}\rangle$

Proof Slide $X$ and resolve as

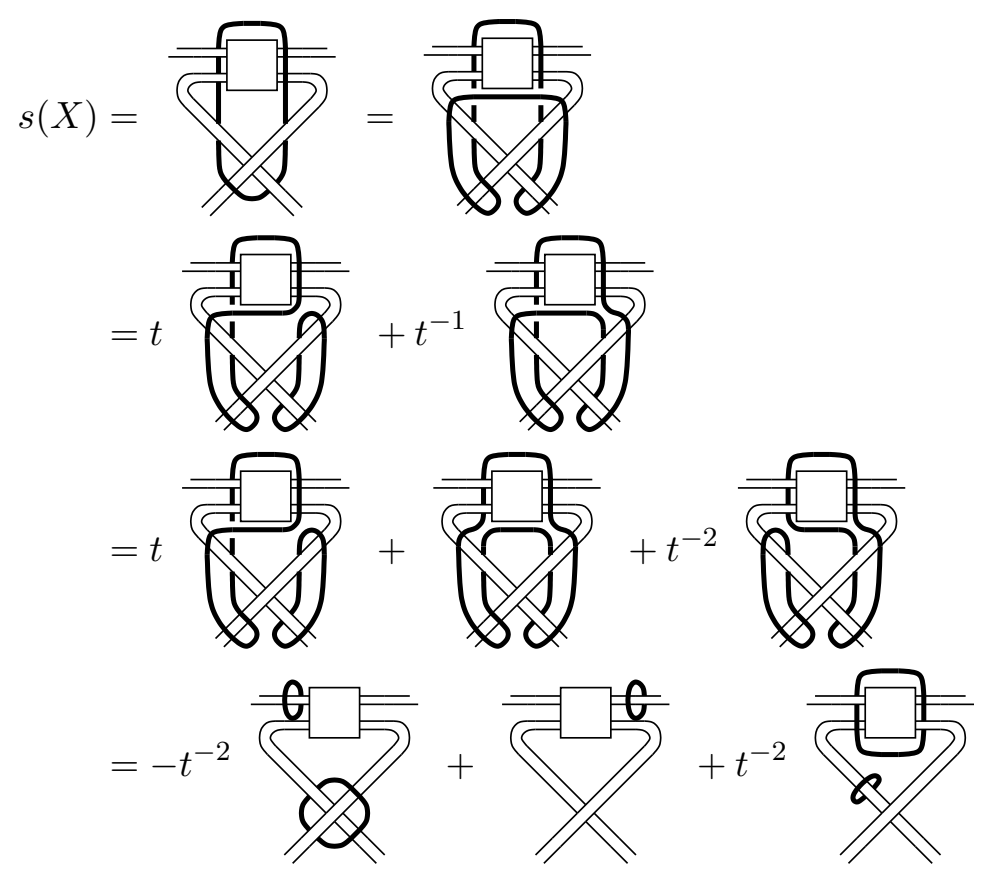

Modulo terms of the form $x L-z L$, this is

$$
s(X)=-t^{-2} x s\left(Y_{q}\right)+c(X)+t^{-2} x c\left(Y_{q}\right)
$$

which is the syzygy $r(X)=-t^{-2} r\left(x Y_{q}\right)$. Inserting $y^{m}$ into the coupon and multiplying by $x^{l} z^{n}$ gives the syzygy

$$
r\left(x^{l} y^{m} z^{n} X\right)=-t^{-2} r\left(x^{l+1} y^{m} z^{n} Y_{q}\right)
$$

Finally, on the right hand side, convert $z$ 's to $x$ 's by repeated applications of

$$
\begin{aligned}
r\left(x^{i} y^{m} z^{k} Y_{q}\right) & =r\left(x^{i} y^{m} z^{k} Y_{q}\right)-r\left(x^{i+1} y^{m} z^{k-1} Y_{q}\right)+r\left(x^{i+1} y^{m} z^{k-1} Y_{q}\right) \\
& =z r\left(x^{i} y^{m} z^{k-1} Y_{q}\right)-x r\left(x^{i} y^{m} z^{k-1} Y_{q}\right)+r\left(x^{i+1} y^{m} z^{k-1} Y_{q}\right)
\end{aligned}
$$

This will express $r\left(x^{l} y^{m} z^{n} Y_{q}\right)$ as $r\left(x^{l+n} y^{m} Y_{q}\right)$ plus terms of the form $x L-z L$. Lemma 5.1 insures that all terms are in $\langle\mathcal{R}\rangle$.

Lemma 5.3 Modulo relations of the form $x L-z L$, we have the syzygy

$$
r\left(y^{m} Y_{q}\right)=t^{4} r\left(y^{m} Y_{q-1}\right)
$$




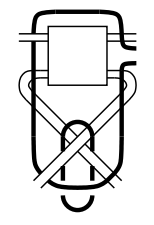

Figure 6

Proof Leaving obvious isotopies to the reader,

$$
t^{4} s\left(Y_{q-1}\right)=-t
$$

Subtract this equation from Equation (3) and insert $y^{m}$ as usual.

Lemma 5.4 For $0 \leq k \leq q, r\left(x^{l} y^{m} Y_{k}\right) \in\langle\mathcal{R}\rangle$.

Proof Induct downward on $k$. If $k=q$ we are looking at $r\left(x^{l} y^{m} Y_{q}\right)$. If $k=q-1$, apply the syzygy from Lemma 5.3 multiplied by $x^{l}$.

If $k \leq q-2$, apply $r$ to Equation (2) $k+1$ twists in the coupon. Modulo terms of the from $x L-z L$, this becomes the syzygy

$$
r\left(y Y_{k+1}\right)=t^{2} r\left(Y_{k}\right)+t^{-2} r\left(Y_{k+2}\right)+r(x X)+r(x Z)
$$

Solve for $r\left(Y_{k}\right)$, multiply by $x^{l}$, and insert $y^{m}$ in the usual place.

Lemma $5.5 r\left(x^{l} y^{m} z^{n} Y_{0}\right) \in\langle\mathcal{R}\rangle$.

Proof Convert $r\left(x^{l} y^{m} z^{l} Y_{0}\right)$ to $r\left(x^{l+n} y^{m} Y_{0}\right)$ as in the proof of Lemma 5.2. Then apply Lemmas 5.1 and 5.4 .

Lemma 5.6 $r\left(x^{l} y^{m} z^{n} U\right) \in\langle\mathcal{R}\rangle$.

Proof Consider the link in Figure 6] The relative component is $Y_{q}$, and the closed component isotops into the coupon where it resolves into some polynomial $p(x, y, z)$. On the other hand, by resolving the crossings as shown we 
obtain (modulo terms of the form $x L-z L$ )

$$
\begin{aligned}
& p Y_{q}=t^{2} U+x \text { = } \\
& =t^{2} U+x \stackrel{t=2}{=} \\
& =t^{2} U+x=
\end{aligned}
$$

Now apply $r$ to this equation to obtain the syzygy

$$
r\left(p Y_{q}\right)=t^{2} r(U) \pm r(x Z)-t^{2} r\left(Y_{0}\right)
$$

(The sign of $r(x Z)$ depends on the number of twists in the coupon.) Insert $y^{m}$, multiply by $x^{l} z^{n}$, and solve for $r(U)$. Except for the term $r\left(x^{l} y^{m} z^{n} U\right)$, convert all $z$ 's to $x$ 's as usual. The resulting linear combination will lie in $\langle\mathcal{R}\rangle$

We have shown that $\langle\mathcal{R}\rangle=r\left(K_{1}(H)\right)$, so $K\left(M_{q}\right)$ must be presented as in Proposition 1.

\section{References}

[1] D Bullock, The $(2, \infty)$-skein module of the complement of a $(2,2 p+1)$ torus knot, J. Knot Theory Ramifications 4 (1995) 619-632 MathReview

[2] D Bullock, On the Kauffman bracket skein module of surgery on a trefoil, Pacific J. Math. 178 (1997) 37-51 MathReview

[3] D Bullock, A finite set of generators for the Kauffman bracket skein algebra, Math. Z. 231 (1999) 91-101 MathReview

[4] D Bullock, Rings of $S L_{2}(\mathbb{C})$-characters and the Kaufman bracket skein module, Comment. Math. Helv. 72 (1997) 521-542 MathReview

[5] D Bullock, C Frohman, J Kania-Bartoszyńska, Understanding the Kaufman bracket skein module, J. Knot Theory Ramifications 8 (1999) 265-277 MathReview

[6] C Frohman, R Gelca, W Lo Faro. The A-polynomial from the noncommutative viewpoint, Trans. Amer. Math. Soc. 354 (2001) 735-747 MathReview

[7] J Hoste, J H Przytycki, The $(2, \infty)$-skein module of lens spaces; a generalization of the Jones polynomial, J. Knot Theory Ramifications 2 (1993) 321-333 MathReview 
[8] J Hoste, J H Przytycki, The Kauffman bracket skein module of $S^{1} \times S^{2}$, Math Z. 220 (1995) 65-73 MathReview

[9] R M Kashaev, The hyperbolic volume of knots from the quantum dilogarithm, Lett. Math. Phys. 39 (1997) 269-275 MathReview

[10] W Lo Faro, A Mayer-Vietoris Theorem for the Kauffman bracket skein module, $\mathrm{PhD}$ thesis, Univ. of Iowa (1998)

[11] W Lo Faro, A Mayer-Vietoris Theorem for the Kauffman bracket skein module, J. Knot Theory Ramifications 8 (1999) 721-729 MathReview

[12] H Murakami, J Murakami, The colored Jones polynomials and the simplicial volume of a knot, Acta Math. 186 (2001) 85-104 MathReview

[13] J H Przytycki, Skein modules of 3-manifolds, Bull. Pol. Acad. Sci. 39 (1991) 91-100 MathReview

[14] J H Przytycki, A Sikora, On skein algebras and $S L_{2}(\mathbb{C})$-character varieties, Topology 39 (2000) 115-148 MathReview

[15] T Takata, The colored Jones polynomial and the A-polynomial for twist knots, arXiv:math.GT/0401068

[16] Y Yokota, From the Jones polynomial to the A-polynomial of hyperbolic knots, Interdisciplinary Inform. Sci. 9 (2003) 11-21 MathReview

Department of Math, Boise State University

Boise, ID 83725, USA

and

Department of Math, University of Wisconsin

Stevens Point, WI 54481, USA

Email: bullock@math.boisestate.edu and Walter.LoFaro@uwsp.edu

Received: 2 February 2004 Revised: 3 February 2005 